\title{
Impact of Phaeobacter inhibens on marine eukaryote-associated microbial communities
}

\author{
Dittmann, Karen K.; Sonnenschein, Eva C.; Egan, Suhelen; Gram, Lone; Bentzon-Tilia, Mikkel
}

Published in:

Environmental Microbiology Reports

Link to article, DOI:

10.1111/1758-2229.12698

Publication date:

2019

Document Version

Peer reviewed version

Link back to DTU Orbit

Citation $(A P A)$ :

Dittmann, K. K., Sonnenschein, E. C., Egan, S., Gram, L., \& Bentzon-Tilia, M. (2019). Impact of Phaeobacter inhibens on marine eukaryote-associated microbial communities. Environmental Microbiology Reports, 11(3), 401-413. https://doi.org/10.1111/1758-2229.12698

\section{General rights}

Copyright and moral rights for the publications made accessible in the public portal are retained by the authors and/or other copyright owners and it is a condition of accessing publications that users recognise and abide by the legal requirements associated with these rights.

- Users may download and print one copy of any publication from the public portal for the purpose of private study or research.

- You may not further distribute the material or use it for any profit-making activity or commercial gain

- You may freely distribute the URL identifying the publication in the public portal 
Originality-Significance Statement: This study demonstrates that the marine, host-associated and conspicuous secondary metabolite producer Phaeobacter inhibens can shape the microbiomes of eukaryotic host organisms. It also offers insight into how the abundance of specific members, such as vibrios, of the microbial communities of the coccolithophorid microalga Emiliania huxleyi and the European flat oyster Ostrea edulis is affected by increasing abundance of P. inhibens.

\footnotetext{
()

Summary

Bacteria-host interactions are universal in nature and have significant effects on host functionality. Bacterial secondary metabolites are believed to play key roles in such interactions as well as in interactions within the host-associated microbial community. Hence, prominent secondary metabolite-producing bacteria may be strong drivers of microbial community composition in natural host-associated microbiomes. This has however not been rigorously tested, and the purpose (1) of this study was to investigate how the secondary metabolite producer Phaeobacter inhibens affects the diversity and composition of microbiomes associated with the microalga Emiliania leyi and the European flat oyster, Ostrea edulis. Roseobacters were indigenous to both communities exhibiting relative abundances between $2.8 \%$ and $7.0 \%$. Addition of P. inhibens Caused substantial changes in the overall structure of the low-complexity microbiome of E. huxleyi, but did not shape microbial community structure to the same degree in the more complex oyster microbiomes. Species-specific interactions occurred in both microbiomes and specifically the abundances of other putative secondary metabolite-producers such as vibrios and pseudoalteromonads were reduced. Thus, the impact of a bioactive strain like $P$. inhibens on hostassociated microbiomes depends on the complexity and composition of the existing microbiome.
} 


\section{Introduction}

In nature, microorganisms live and interact as part of complex multispecies communities. These interspecies interactions may be of a synergistic, amensal, or commensal nature and can be facilitated by the exchange of metabolites in syntrophic cooperation, or by the production of bioactive secondary metabolites (Cole, 1982). In parallel to the use of secondary metabolites as

defense against microbial infections by plants (Pusztahelyi et al., 2015), bacterial secondary metabolites with antimicrobial properties are currently believed to facilitate the success of the compound-producer by killing competitors. Not all bacteria are equally proficient in secondary metabolite production, and whereas some groups appear to produce few or no metabolites, others such as filamentous soil bacteria and marine vibrios, roseobacters, and Pseudoalteromonas spp. produce an array of different bioactive compounds (Brinkhoff et al., 2004; Murphy et al., 2012; Rasmussen et al., 2014; Maansson et al., 2016; Sonnenschein, Stierhof, et al., 2017). Hence, if the

role of these compounds is to eliminate competing microorganisms, proficient secondary metabolite producers should be strong drivers of microbial community composition in natural environments.

The Roseobacter group represents one of the most abundant groups of marine bacteria, constituting on average $3-5 \%$ of microbial communities in the upper mixed layer (Wietz et al., 2010). On a global scale, the group exhibits a positive abundance-chlorophyll $a$ correlation (Wietz et al., 2010) and may exhibit relative abundances of up to 20 - $30 \%$ during algal blooms (González and Moran, 1997; González et al., 2000; Zubkov et al., 2001; West et al., 2008), suggesting association with microalgae. The paraphyletic Roseobacter group comprises multiple deeply branching clades

(Newton et al., 2010; Simon et al., 2017), of which especially clade 1 includes prominent producers of bioactive secondary metabolites, such as the antimicrobial compounds tropodithietic acid (TDA) (Brinkhoff et al., 2004; Bruhn et al., 2005; Sonnenschein, Nielsen, et al., 2017), indigoidine (Cude 
et al., 2012; Gromek et al., 2016), and likely multiple other small molecules (Machado et al., 2015; Bentzon-Tilia and Gram, 2017; Sonnenschein et al., 2018).

One conspicuous genus of Roseobacter clade 1 is Phaeobacter, which is often found in microbial Communities associated with a wide variety of marine eukaryotes including micro- and macroalgae (Rao et al., 2005; Segev, Wyche, et al., 2016), mesozooplankton (Freese et al., 2017), and larger animals such as bivalve molluscs (Ruiz-Ponte et al., 1998; Prado et al., 2009; Wegner et al., 2013). Recently, it was shown that the species Phaeobacter inhibens produces small bioactive molecules, e.g. indole-3-acetic acid, which affect the metabolism of the coccolithophorid microalga Emiliania huxleyi (Segev, Castañeda, et al., 2016; Segev, Wyche, et al., 2016). It has been proposed that the interaction between E. huxleyi and $P$. inhibens exhibits a biphasic pattern where a mutualistic symbiosis gives way for a parasitic interaction where the bacteria accelerates algal lysis in response to algal break-down products (Seyedsayamdost et al., 2011). Mutualistic mechanisms have also been suggested for the symbiosis between bioactive Roseobacter clade 1 organisms living in association with the Hawaiian bobtail squid (Euprymna scolopes), specifically on the outer surface of the eggs and in the accessory nidamental gland (Collins et al., 2012; Gromek et al., 2016), where they supposedly ward off potential pathogens through the production of antimicrobials. Hence, proficient secondary metabolite-producing species, such as members of the Phaeobacter genus might be strong modulators of both the behavior and the microbiome composition of their eukaryotic hosts.

For $P$. inhibens, TDA is the most studied secondary metabolite and its antimicrobial property is likely a result of the ability of TDA to act as a proton antiporter at the cytoplasmic membrane (Wilson et al., 2016). Hence, TDA is a broad-spectrum antibiotic affecting a wide range of both Gram-positive and Gram-negative bacteria (Porsby et al., 2011). Despite the fact that resistance towards TDA does not arise easily (Porsby et al., 2011; Rasmussen et al., 2016), a large fraction of 
bacterial isolates from eukaryote-associated microbiomes similar to those harboring TDA-producers are tolerant towards TDA (Harrington et al., 2014). Such microbiomes may hence be resilient to perturbations caused by compounds such as TDA. Recently, Geng et al. (2016) showed that additions of the pure TDA compound had pronounced dose-dependent effects on community structure and composition of the microalgal Nannochloropsis salina microbiome at relatively low concentrations $(31-500 \mathrm{nM})$. At these concentrations, TDA may act as an inter-microbial signaling molecule rather than an antibiotic (Beyersmann et al., 2017). However, the concentrations, at which secondary metabolites are produced in natural communities are currently unknown, and to what extent the presence of secondary metabolite-producing organisms directly affect the microbial communities has not been addressed. Hence, considering the scarcity of in vivo models exploring the effects of prominent secondary metabolite producers on natural eukaryote-associated microbiomes, the purpose of the present study was to establish co-culture model systems to investigate how TDA-producing $P$. inhibens shapes the E. huxleyi and Ostrea edulis microbiomes over time. One of our key goals was to investigate how different concentrations of TDA-producing $P$. inhibens would alter the community structure due to a gradual increase in roseobacters (doseponse) mimicking the increase in algal blooms. The algal model system was chosen as there is a marked increase in roseobacters in the natural environment during algal blooms and we used levels of P. inhibens DSM 17395 reflecting the in situ abundances (Amin et al., 2015; Segev, Wyche, et al., 2016; Sonnenschein et al., 2018). The oyster system was chosen as a model for another trophic layer, which would be affected by algal blooms and potentially be exposed to high densities of roseobacters given its feeding mechanism. 


\section{Results}

The impact of $P$. inhibens strain DSM 17395 on marine eukaryote-associated microbiomes was assessed by sequencing $16 \mathrm{~S}$ rRNA gene V4 region amplicons from the two different hosts; $E$. huxleyi (microalga) and O. edulis (European flat oyster).

\section{Emiliania huxleyi-associated microbial community composition.}

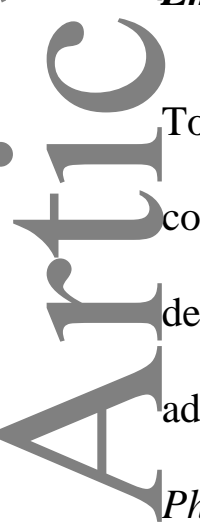

To investigate if $P$. inhibens can affect the microbiome composition and diversity of microalgae, co-cultures of E. huxleyi and $P$. inhibens were set up in three groups; i) untreated controls, ii) low density $\left(10^{4} \mathrm{CFU} \mathrm{mL} \mathrm{m}^{-1}\right)$ of $P$. inhibens, and iii) high density $\left(10^{6} \mathrm{CFU} \mathrm{mL}^{-1}\right)$ of $P$. inhibens. The added densities were equivalent to 0.4 and 40 bacterial cells per algal cell, and 0.09 and 9 Phaeobacter cells per bacterium of the initial microbiome, mimicking the ratios observed during algal blooms. The initial algal microbiome was sampled at time point $0 \mathrm{~h}$ prior to treatment and the total bacterial count was $5.48 \pm 0.58 \log _{10} \mathrm{CFU} / \mathrm{mL}$ (using a qPCR-based quantification). Each coculture was sampled three times: at $24 \mathrm{~h}, 48 \mathrm{~h}$ and $96 \mathrm{~h}$. The most abundant bacterial OTU in the co-cultures (EH_OTU 4) was identified as a Phaeobacter sp. (SILVA annotation) and the resentative sequence was $100 \%$ identical to P. inhibens strain DSM 17395 (accession no. CP002976.1). However, it is important to note that the V4 region does not allow for the discrimination between $P$. inhibens and closely related roseobacters (Table S1). In the amplicon sequencing data, EH_OTU 4 was observed in all samples at relative abundances of $4.0 \%$ to $4.2 \%$ in the initial microbiome, $4.2 \%$ to $8.2 \%$ in the untreated controls ( $24 \mathrm{~h}$ to $96 \mathrm{~h}$ ), $8.3 \%$ to $11.2 \%$ in the microbiomes treated with the low density of P. inhibens, and finally $53.8 \%$ to $79.9 \%$ in the microbiomes of algae treated with the high density. However, using qPCR, P. inhibens was below the detection limit $\left(3.06 \log _{10} \mathrm{CFU} / \mathrm{mL}\right)$ in the initial microbiome samples. Accordingly, EH_OTU 4 was excluded from subsequent analyses. Taxonomy and relative abundance of the ten most 
abundant OTUs (excluding EH_OTU 4) across all samples can be found in Supplementary material (Table S2, Table S3).

The community was dominated by orders of the Proteobacteria and Bacteroidetes phyla regardless

of treatment or incubation time (Figure 1) and neither parameter influenced the composition at class or order level notably. Gammaproteobacteria, Flavobacteria, and Alphaproteobacteria were equally dominating across samples. Co-culturing with high levels of $P$. inhibens altered the relative abundance of other Rhodobacterales members from $18 \%$ to $23 \%$ in controls, to $16 \%$ to $24 \%$ in low $P$. inhibens density co-cultures, and $7.1 \%$ to $9.7 \%$ in high $P$. inhibens density co-cultures except for one high-density, $96 \mathrm{~h}$ co-culture (19\%; Figure 1). However, the relative abundance of the order Flavobacteriales and an unidentified Gammaproteobacteria order remained stable across treatments with relative abundances of $23.1 \%$ to $50.1 \%$ and $22.0 \%$ to $34.8 \%$, respectively.

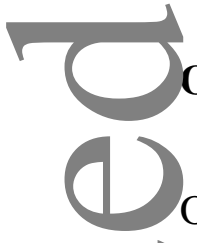

\section{Oyster-associated microbial community composition}

Oysters were divided into two groups: i) untreated controls, and ii) high density $\left(10^{7} \mathrm{CFU} \mathrm{mL} \mathrm{m}^{-1}\right)$ of P. inhibens. Two to three oysters were sacrificed before $(0 \mathrm{~h})$ and $48 \mathrm{~h}$ after treatment to assess ential changes occurring in the microbiome. The total bacterial count of the initial microbiome was $6.99 \pm 0.91 \log _{10} \mathrm{CFU} / \mathrm{mL}$ (using qPCR-based quantification), hence, the density of $P$. inhibens is equivalent to 0.3 Phaeobacter cells to 1 indigenous bacterium. The most abundant OTU in the more complex oyster microbiomes (OE_OTU 1) was identified as an unclassified member of the Rhodobacteraceae family (SILVA annotation). The OE_OTU 1 representative sequence was $100 \%$ identical to P. inhibens strain DSM 17395 (accession no. CP002976.1) (Table S4). OE_OTU 1 was observed in all samples; the relative abundance accounted for $2.8 \%-7.0 \%$ of the initial microbiome, decreased to $0.5 \%-0.6 \%$ upon 48 hour incubation of the untreated control oysters but was increased to $33.1 \%-46.7 \%$ in the microbiomes treated with $P$. inhibens. However, $P$. 
inhibens was below the qPCR detection limit $\left(3.06 \log _{10} \mathrm{CFU} / \mathrm{mL}\right)$ in the initial microbiome samples. Accordingly, OE_OTU1 was excluded from subsequent analyses. Taxonomy and relative abundance of the ten most abundant OTUs (excluding OE_OTU 1) across all samples can be found in Supplementary material (Table S5, Table S6).

Five different phyla (Bacteroidetes, Firmicutes, Fusobacteria, Proteobacteria, and Tenericutes) with relative abundances above $2 \%$ were observed across the samples of the oyster microbiomes.

Proteobacteria was the dominant phylum in all samples and it increased in relative abundance from 0 to 48 hours. At class level, Gammaproteobacteria was the major contributor, which was mainly due to the orders Alteromonadales and Vibrionales (Figure 2). The relative abundance of Vibrionales decreased in oysters treated with P. inhibens $(3.1 \%-4.4 \%)$ compared to the $48 \mathrm{~h}$ control oysters $(16 \%-29 \%)$. In contrast, Alteromonadales were more abundant in the P. inhibens treated samples $(56 \%-70 \%)$ in comparison to the control $(31 \%-4$
dominant in the untreated controls after $48 \mathrm{~h}$, but not in the samples tr
Impact on richness and diversity of host-associated microbiomes

Microbiomes associated with the algae were less complex than the oyster microbiomes exhibiting Cestimated OTU richness values (Chao1) in the range of 140 to 493 OTUs (Figure 3A) compared to 810 to 5,746 OTUs for oysters (Figure 3B). Some variation was observed in the estimated richness of the E. huxleyi microbiomes, but no clear temporal patterns or treatment effects were apparent. In contrast, the $P$. inhibens treatment of oysters resulted in a significantly increased richness index in comparison to the initial and the control populations (t-test, $\mathrm{P}<0.05$ ), while the latter, untreated microbiomes ( $0 \mathrm{~h}$ vs. $48 \mathrm{~h}$ ) did not significantly change during the time of incubation (t-test, $\mathrm{P}>$ 0.05). As indicated by the estimated richness of the microbiomes, the diversity of oyster 
microbiomes (Figure S2B) were higher than that of the alga (Figure S2A), but in contrast to the effect on species richness in the oyster microbiome, the introduction of $P$. inhibens did not affect overall diversity in any of the microbiomes (Figure S2). Hence, the addition of $P$. inhibens had a significant positive effect on species richness in the oyster microbiomes, but the abundances of these species were not evenly distributed and did not affect overall microbiome diversity.

\section{Impact on community structure of host-associated microbiomes}

Community structure analyses of both E. huxleyi and O. edulis microbiomes were based on Bray2 Curtis dissimilarity indexes. For E. huxleyi, treatment-dependent clustering was observed for the microbiomes receiving the high density of $P$. inhibens, and as indicated by order-level community composition (Figure 1), the untreated communities clustered independently of sampling time point (Figure 4), and hence the effect of $P$. inhibens on the community was immediate (within $0-24 \mathrm{~h}$ ). In agreement with the negligible effects on the abundance of EH_OTU 4 in communities receiving C the low density, these communities were interspersed between the other treatment groups. Furthermore, samples from replicate cultures of the same treatment were found in separate, individual clusters regardless of time point, thus indicating a strong "bottle" effect in the low density co-cultures (Figure 4). Similarly, for O. edulis, the microbiomes co-cultured with the high P. inhibens densities clustered separately from the untreated controls (Figure 5) and the untreated ( mici microbiomes were dispersed independent of time point, suggesting that high levels of $P$. inhibens altered community structure for both microbiomes. However, there was substantial variation between some biological replicates.

Analysis of Molecular Variance (AMOVA; Bray Curtis distances; 10,000 iterations) on the $O$. edulis microbiomes indicated a significant variance among the three treatment groups $\left(\mathrm{T}_{0}, 48 \mathrm{~h}\right.$ control, $48 \mathrm{~h} P$. inhibens treatment; $\mathrm{P}=0.0044)$, but none of the pairwise variance comparisons 
were significantly different from each other. AMOVA was not performed on the E. huxleyi microbiome given the low replication level. However, the analyses of the community structures indicate that the less complex microbiome associated with E. huxleyi was likely influenced by the introduction of high concentrations of $P$. inhibens, while the differences observed in the more complex oyster microbiome were not significant under the conditions tested.

\section{Impact on individual OTUs}

At the order level, the effect of $P$. inhibens on community composition in the E. huxleyi microbiome was subtle (Figure 1), and hence we investigated how the differences observed in community structure came across at the species level (OTU clustering at $97 \%$ sequence similarity). Analyzing the 40 most abundant OTUs from both host-associated microbiomes confirmed some variation among replicates. For the E. huxleyi microbiome, the relative abundances of the seven most abundant OTUs $(2,3,5,6,7,10,8$; Table S2) were unaffected by the presence of $P$. inhibens (Figure 4). EH_OTU 9 (Loktanella sp.) and EH_OTU 12 (Marivita sp.) decreased only in communities treated with the high density of $P$. inhibens. Two gammaproteobacterial OTUs of the Vibrio and Pseudoalteromonas genera (EH_OTU 20 and 18) were present in the initial microbiomes and increased in relative abundance over time in control microbiomes. However, their abundance decreased in microbiomes supplemented with $P$. inhibens, irrespective of treatment density. In contrast, two OTUs, EH_OTU 33 (Colwellia sp.) and 56 (Neptuniibacter sp.), were abundant in the microbiomes co-cultured with $P$. inhibens while absent in the controls throughout the experiment. Thus, trends of co-occurring changes in relative abundance in the presence or absence of $P$. inhibens seems to be species if not strain specific in the E. huxleyi microbiome.

In contrast to observations from the E. huxleyi microbiome, OTUs related to Colwellia (OE_OTU 2 and 6; Table S5) were unaffected by the presence of $P$. inhibens in the oyster microbiome (Figure 
5). However, as for the E. huxleyi microbiome, species of the Vibrio and Pseudoalteromonas genera (OE_OTU 3 and 4) decreased in relative abundance in samples treated with $P$. inhibens. Furthermore, OE_OTUs 7, 9, 26 (Mycoplasma sp.), 5 (Shewanella sp.), and 77 (unclassified proteobacterial OTU) also decreased in the presence of P. inhibens. Finally, one Mycoplasmarelated OTU (OE_OTU 729) increased in relative abundance in the presence of $P$. inhibens. Henceforth, strain rather than species-specific changes in relative abundances of Mycoplasma species occur in the presence of $P$. inhibens in the oyster microbiome.

To investigate a potentially specific, amensal interaction between $P$. inhibens and vibrios, given the potential pathogenicity of certain species, we assessed Vibrio spp. abundances in oyster microbiomes quantitatively using quantitative real-time PCR (qPCR). As suggested by the community composition analysis (Figure 2), the introduction of $P$. inhibens did not remove vibrios in the oyster microbiomes completely (Figure S3). However, it resulted in a reduction of Vibrio spp. 16S rRNA genes to $5.61 \pm 0.23 \log _{10}$ copies $\mathrm{g}^{-1}$ as compared to the initial abundance of $5.91 \pm 0.40$ $\log _{10}$ copies $\mathrm{g}^{-1}$, and it further reduced Vibrio abundances significantly with more than one order of magnitude compared to control oysters $(6.89 \pm 0.18 ; \mathrm{P}=0.0328)$, thus corroborating the results of the sequence analyses.

$\circlearrowright$

\section{Discussion}

Bacterial communities associated with eukaryotes have a significant impact on the health and function of their hosts, and investigating how microbiomes of higher organisms are formed and affected by external and internal factors, has become an area of broad and current interest (e.g. Ley et al., 2008; Lebeis et al., 2015). Some members of the host-associated microbiomes carry the capacity to produce bioactive secondary metabolites that may act in the competition with other 
members of the microbiomes, yet, it is currently unknown to what extent these bacteria can directly influence and shape the structure of host-associated microbial communities. Our results suggest that the TDA-producing $P$. inhibens has the capacity to influence and shape marine eukaryoteassociated microbiomes, yet the effects are variable, dependent on the abundance of $P$. inhibens, and on the complexity and species composition of the host microbiome. Furthermore, the imposed changes occur within a short temporal scale $(\leq 24 \mathrm{~h})$ and are otherwise independent of time (within five days).

The two eukaryote model systems were used to investigate how a TDA-producing $P$. inhibens would shape eukaryote-associated microbiomes; during algal blooms vs. non-bloom conditions for the microalga, and how potentially high densities of roseobacters would impact the microbiome of a filter-feeder at another trophic layer during algal blooms. Several studies have been conducted on bacteria associated with E. huxleyi and oysters (Zabeti et al., 2010; Carella et al., 2013; Farto Seguín et al., 2014; Green et al., 2015), but the amount of comprehensive culture-independent, diversity studies is limited. The oyster microbiomes of Crassostrea spp. have been characterized (King et al., 2012; Wegner et al., 2013; Ossai et al., 2017; Vezzulli et al., 2018) due to their importance in aquaculture, but the present study is to the best of our knowledge, the first cultureindependent study investigating the bacterial population of $O$. edulis. Roseobacters were indigenous to both of the investigated microbiomes in agreement with previous findings of molluscan species (Ruiz-Ponte et al., 1998; Grigioni et al., 2000; Barbieri et al., 2001; Martens et al., 2006; Prado et al., 2009) and algae (González et al., 2000; Green et al., 2015; Segev, Wyche, et al., 2016). The occurrence of roseobacters in both native microbiomes supports our choice of $P$. inhibens DSM 17395 as a model organism for a secondary metabolite producer in the natural environment of the eukaryotes in the event of an algal bloom. 
The estimated OTU richness of the E. huxleyi microbiome was four to seven times lower than the richness of the complex $O$. edulis microbiome. Increasing the $P$. inhibens abundance in the low complexity microbiome of E. huxleyi had little to no effect on the total species richness and diversity, whereas the richness increased dramatically in oysters. If $P$. inhibens uses its bioactive compounds to kill competitors, i.e. as antibiotics, a decrease in bacterial richness and diversity could be expected, but the addition of $P$. inhibens had the opposite effect in the oyster system, indicating that the bioactive compounds, such as TDA, are either not produced or serve another function. The expression of TDA-encoding genes have been shown in algal co-culture systems (D’Alvise et al., 2012). Furthermore, TDA is likely produced in our model systems as $P$. inhibens DSM 17395 was pre-grown at conditions known to induce TDA production, i.e. nutrient/iron rich broth. While nutrient-rich medium, such as marine broth, differs from the natural environment, some heterotrophs thrive at high nutrient levels (Alonso and Pernthaler, 2006; Pohlner et al., 2017), which are comparable to the dense, nutrient-rich surroundings of algal cells or in oysters.

Henceforth, it is most likely that TDA has another function than being a broad-spectrum defense compound, and that it is probably highly dependent on the investigated conditions.

Snecies-rich microbiomes are typical of healthy, marine invertebrates including oysters (King et al., 2012; Wegner et al., 2013), whereas ill and diseased oyster microbiomes are characterized by a decrease in complexity, loss of rare bacterial strains (Wegner et al., 2013), disruption of the community structure (Lokmer and Mathias Wegner, 2015), and increased abundance of few, specialist OTUs (Wegner et al., 2013; Lokmer and Mathias Wegner, 2015). Thus, it could be speculated that an increase in $P$. inhibens might be beneficial for the host by decreasing the load of potential opportunistic pathogens and allowing rare taxa to proliferate, which has been suggested as the role of various Roseobacter group members associated with other molluscs (Collins et al., 2012; Gromek et al., 2016) and microalgae (Seyedsayamdost et al., 2011). 
P. inhibens influenced the bacterial community structure in a dose dependent manner, independently of temporal space. The high density of $P$. inhibens caused a shift in the community structure of the E. huxleyi microbiome, but no evident clustering patterns were observed for the algal microbiome exposed to the low density. While due to the low sample number these results still require further verification, similar patterns were observed in the microbiome structure of $N$. salina after the addition of pure TDA (Geng et al., 2016).

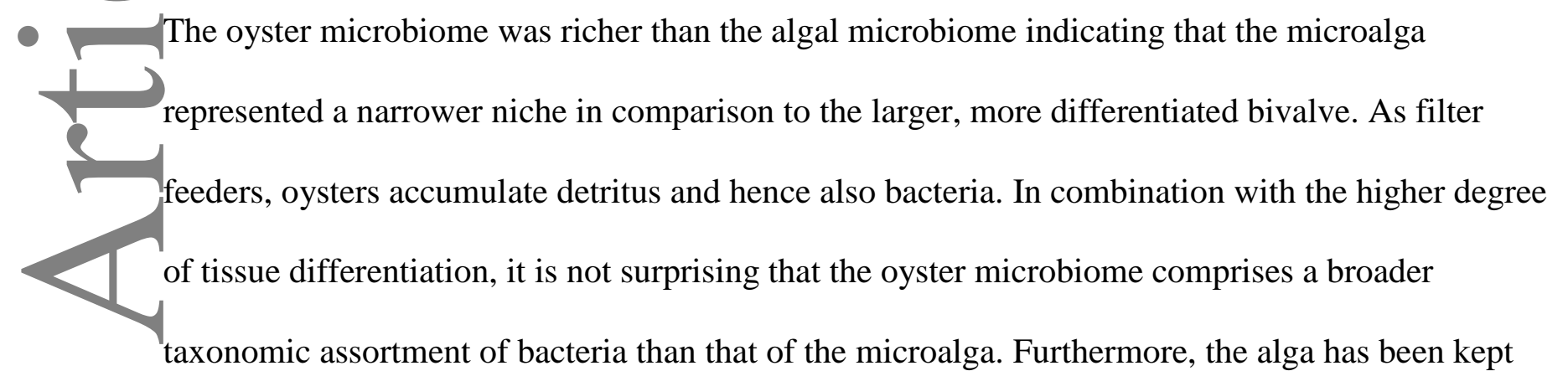
under continuous laboratory cultivation since its isolation, which may have reduced the richness and diversity of its associated microbiome. Proteobacteria dominated the bacterial community of both $E$. huxleyi and O. edulis in agreement with previous observations in coccolithophorid microalgae and other oyster species (Wegner et al., 2013; Green et al., 2015; García Bernal et al., 2017). Green et al., 2015 used a culture-dependent approach and found that Alphaproteobacteria were dominating in their coccolithophorid cultures. Similarly, it has been reported that Gamma- and Alphaproteobacteria of the orders Alteromonadales and Rhodobacterales dominated E. huxleyi blooms in the North Atlantic Ocean (Segev, Wyche, et al., 2016). In our culture-independent approach, we found both Gamma- and Alphaproteobacteria as well as Flavobacteria (Bacteroidetes) dominated in the E. huxleyi microbiomes.

In O. edulis, Tenericutes and Bacteroidetes were prominent phyla although less dominating than Proteobacteria. Wegner et al. (Wegner et al., 2013) similarly found Proteobacteria as the dominant phyla followed by Flavobacteria and Bacteroidetes in pacific oysters (Crassostrea gigas). The 
community composition and dominance of individual taxa depends on local environment, tissue (different organs vs. whole organism), and individual oyster genetics and physiology (King et al., 2012; Wegner et al., 2013; García Bernal et al., 2017; Vezzulli et al., 2018). The Vibrionales and Mycoplasmatales orders decreased in abundance in the presence of $P$. inhibens while Alteromonadales increased in abundance. In the microbiome of the microalga N. salina, the abundance of Alteromonadales also increased in the presence of pure TDA while Rhodobacteraceae decreased (Geng et al., 2016). We did not find that $P$. inhibens decreased other Rhodobacterales in the oyster microbiome, however, any change in relative abundance of this order would not be 8 observed since it was below the $2 \%$ cutoff in all microbiomes. Although, the relative abundance of the Rhodobacterales order did decrease in the E. huxleyi microbiome when exposed to the high density of $P$. inhibens while the abundance of Alteromonadales was unaffected. Hence, the impact of the TDA-producer $P$. inhibens on overall community composition is likely dependent on the eukaryotic host and/or the indigenous community composition.

\section{(1)}

Given the subtle alterations at higher taxonomic levels in some microbiomes, we assessed changes at the OTU-level (species level) to identify the underlying causes. Indeed, we observed some (4) differences in the OTUs affected by different densities of $P$. inhibens, though with noticeable variability between replicate cultures. Some species (Colwellia and Sulfurospirillum) were either unaffected or increased by the presence of P. inhibens, whereas others including Vibrio spp. and Pseudoalteromonas spp. were reduced in both eukaryote microbiomes. Interestingly, vibrios and pseudalteromonads are also considered proficient secondary metabolite producers and hence it is plausible that $P$. inhibens specifically and efficiently antagonizes other microorganisms occupying similar niches. The efficient inhibition of vibrios by TDA-producing roseobacters have been repeatedly demonstrated in studies targeting the fish pathogenic Vibrio spp. in marine eukaryotes (Porsby et al., 2008; Prado et al., 2009; D’Alvise et al., 2012; Grotkjær, Bentzon-Tilia, Paul W 
D’Alvise, et al., 2016; Grotkjær, Bentzon-Tilia, Paul W. D’Alvise, et al., 2016; Porsby and Gram, 2016; Rasmussen et al., 2018). The high degree of target organism specificity is however somewhat surprising considering the spectrum of bioactivity of TDA (Porsby et al., 2011). Multiple Mycoplasma spp. decreased in abundance while a single OTU classified to the same genus increased in abundance further substantiating that the effects of $P$. inhibens are very specific (species level or below). Corroborating the observation that the majority of the species in the microbiomes exhibited minor changes in abundance due to the presence of $P$. inhibens, is the

findings by Harrington et al. (2014) who observed a high degree of TDA tolerance in non-TDA producing bacterial isolates from marine eukaryote-associated microbiomes. Thus, the impact of increased abundances of a particular secondary metabolite producing microorganism such as $P$. inhibens seems to be highly selective and likely dependent on its specific secondary metabolite profile.

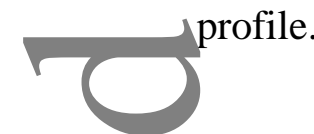

( In conclusion, TDA-producing $P$. inhibens can shape host-associated microbiomes of marine eukaryotes. These alterations are subtle at the broader taxonomic levels, but seems to be highly selective and consistent at the OTU level (97\% similarity) across eukaryote host systems. The . effects of $P$. inhibens on the species richness and microbiome structure are multifaceted; the richness in the complex microbiome of oysters increased while it remained constant in the microalgal model and the microbiome structure shifted only due to high densities of $P$. inhibens. Thus, the impact likely relies on the composition and complexity of the indigenous bacterial communities. This suggests that a highly diverse microbiome is more stable, though future work across more, varied eukaryote-microbiome associations with larger sample sizes would provide more knowledge to substantiate this hypothesis.

\section{Acknowledgements}


The authors wish to thank Professor Torsten Thomas for providing valuable discussions. We acknowledge the help by Cisse Hedegaard Porsby and the Danish Shellfish Center (National Institute of Aquatic Resources, Technical University of Denmark, Denmark) with providing oysters and setting up experiments with oysters.

The present study was funded by the Technical University of Denmark (PhD grant for KKD), the Villum Kann Rasmussen foundation (grant VKR023285), the Danish Council for Strategic Research, Committee for Strategic Research in Health, Food and Welfare (grant 0603-00515B), the Danish National Research Foundation (grant DNRF137), the Oticon foundation (Personal travel grant for KKD), IDAs og Berg-Nielsens Studie- og Støttefond (Personal travel grant for KKD), the Augustinus foundation (Personal travel grant for KKD), the Fabrikant P.A. Fiskers foundation (Personal travel grant for KKD), and the scholarship Christian og Ottilia Brorsons Rejselegat for yngre videnskabsmænd og -kvinder (Personal travel grant for KKD).

The authors declare no conflict of interests.

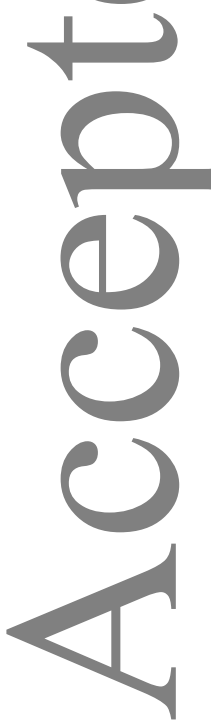




\section{References}

Alonso, C. and Pernthaler, J. (2006) Roseobacter and SAR11 dominate microbial glucose uptake in coastal North Sea waters. Environ. Microbiol. 8: 2022-2030.

Amin, S.A., Hmelo, L.R., van Tol, H.M., Durham, B.P., Carlson, L.T., Heal, K.R., et al. (2015) Interaction and signalling between a cosmopolitan phytoplankton and associated bacteria. Nature 522: 98-101.

Barbieri, E., Paster, B.J., Hughes, D., Zurek, L., Moser, D.P., Teske, A., and Sogin, M.L. (2001) Phylogenetic characterization of epibiotic bacteria in the accessory nidamental gland and egg capsules of the squid Loligo pealei (Cephalopoda: Loliginidae). Environ. Microbiol. 3: 151167.

Bentzon-Tilia, M. and Gram, L. (2017) Biotechnological Applications of the Roseobacter Clade. In, Paterson,R. and Lima,N. (eds), Biotechnological Applications of the Roseobacter Clade. Springer International Publishing, pp. 137-166.

Beyersmann, P.G., Tomasch, J., Son, K., Stocker, R., Göker, M., Wagner-Döbler, I., et al. (2017) Dual function of tropodithietic acid as antibiotic and signaling molecule in global gene regulation of the probiotic bacterium Phaeobacter inhibens. Sci. Rep. 7: 730.

Brinkhoff, T., Bach, G., Heidorn, T., Liang, L., Schlingloff, A., and Simon, M. (2004) Antibiotic Production by a Roseobacter Clade-Affiliated Species from the German Wadden Sea and Its Antagonistic Effects on Indigenous Isolates. Appl. Environ. Microbiol. 70: 2560-2565.

Bruhn, J.B., Nielsen, K.F., Hjelm, M., Hansen, M., Bresciani, J., Schulz, S., and Gram, L. (2005) Ecology, inhibitory activity, and morphogenesis of a marine antagonistic bacterium belonging to the Roseobacter clade. Appl. Environ. Microbiol. 71: 7263-7270. 
Carella, F., Carrasco, N., Andree, K.B., Lacuesta, B., Furones, D., and De Vico, G. (2013) Nocardiosis in Mediterranean bivalves: First detection of Nocardia crassostreae in a new host Mytilus galloprovincialis and in Ostrea edulis from the Gulf of Naples (Italy). J. Invertebr. Pathol. 114: 324-328.

\section{(1)}

Cole, J.J. (1982) Interactions Between Bacteria and Algae in Aquatic Ecosystems. Annu. Rev. Ecol. Syst. 13: 291-314.

Collins, A.J., LaBarre, B.A., Wong Won, B.S., Shah, M. V., Heng, S., Choudhury, M.H., et al. (2012) Diversity and Partitioning of Bacterial Populations within the Accessory Nidamental Gland of the Squid Euprymna scolopes. Appl. Environ. Microbiol. 78: 4200-4208.

Cude, W.N., Mooney, J., Tavanaei, A.A., Hadden, M.K., Frank, A.M., Gulvik, C.A., et al. (2012) Production of the Antimicrobial Secondary Metabolite Indigoidine Contributes to Competitive Surface Colonization by the Marine Roseobacter Phaeobacter sp. strain Y4I. Appl. Environ. Microbiol. 78: 4771-4780.

D’Alvise, P.W., Lillebø, S., Prol-Garcia, M.J., Wergeland, H.I., Nielsen, K.F., Bergh, Ø., and Gram, L. (2012) Phaeobacter gallaeciensis reduces Vibrio anguillarum in cultures of microalgae and rotifers, and prevents vibriosis in cod larvae. PLoS One 7: e43996.

Farto Seguín, R.M., Bermúdez, M.B., Rivera, L., and Nieto, T.P. (2014) Increased Survival of Juvenile Turbot Scophthalmus maximus by Using Bacteria Associated with Cultured Oysters. J. Aquat. Anim. Health 26: 251-262.

Freese, H.M., Methner, A., and Overmann, J. (2017) Adaptation of Surface-Associated Bacteria to the Open Ocean: A Genomically Distinct Subpopulation of Phaeobacter gallaeciensis Colonizes Pacific Mesozooplankton. Front. Microbiol. 8: 1-12. 
García Bernal, M., Trabal Fernández, N., Saucedo Lastra, P.E., Medina Marrero, R., and MazónSuástegui, J.M. (2017) Streptomyces effect on the bacterial microbiota associated to Crassostrea sikamea oyster. J. Appl. Microbiol. 122: 601-614.

Geng, H., Tran-Gyamfi, M.B., Lane, T.W., Sale, K.L., and Yu, E.T. (2016) Changes in the Structure of the Microbial Community Associated with Nannochloropsis salina following Treatments with Antibiotics and Bioactive Compounds. Front. Microbiol. 7: 1155.

González, J.M. and Moran, M.A. (1997) Numerical dominance of a group of marine bacteria in the alpha-subclass of the class Proteobacteria in coastal seawater. Appl. Environ. Microbiol. 63: $4237-42$.

González, J.M., Simó, R., Massana, R., Covert, J.S., Casamayor, E.O., Pedrós-Alió, C., and Moran, M.A. (2000) Bacterial community structure associated with a dimethylsulfoniopropionateproducing North Atlantic algal bloom. Appl. Environ. Microbiol. 66: 4237-46.

(1)

Green, D.H., Echavarri-Bravo, V., Brennan, D., and Hart, M.C. (2015) Bacterial Diversity Associated with the Coccolithophorid Algae Emiliania huxleyi and Coccolithus pelagicus $\mathrm{f}$. braarudii. Biomed Res. Int. 2015: 1-15.

Grigioni, S., Boucher-Rodoni, R., Demarta, A., Tonolla, M., and Peduzzi, R. (2000) Phylogenetic characterisation of bacterial symbionts in the accessory nidamental glands of the sepioid Sepia officinalis (Cephalopoda: Decapoda). Mar. Biol. 136: 217-222.

Gromek, S.M., Suria, A.M., Fullmer, M.S., Garcia, J.L., Gogarten, J.P., Nyholm, S. V., and Balunas, M.J. (2016) Leisingera sp. JC1, a bacterial isolate from hawaiian bobtail squid eggs, produces indigoidine and differentially inhibits vibrios. Front. Microbiol. 7: 1-16.

Grotkjær, T., Bentzon-Tilia, M., D’Alvise, P.W., Dierckens, K., Bossier, P., and Gram, L. (2016) 
Phaeobacter inhibens as probiotic bacteria in non-axenic Artemia and algae cultures. Aquaculture 462: 64-69.

Grotkjær, T., Bentzon-Tilia, M., D’Alvise, P.W., Dourala, N., Nielsen, K.F., and Gram, L. (2016) Isolation of TDA-producing Phaeobacter strains from sea bass larval rearing units and their probiotic effect against pathogenic Vibrio spp. in Artemia cultures. Syst. Appl. Microbiol. 39: $180-188$.

Harrington, C., Reen, F., Mooij, M., Stewart, F., Chabot, J.-B., Guerra, A., et al. (2014) Characterisation of Non-Autoinducing Tropodithietic Acid (TDA) Production from Marine Sponge Pseudovibrio Species. Mar. Drugs 12: 5960-5978.

King, G.M., Judd, C., Kuske, C.R., and Smith, C. (2012) Analysis of Stomach and Gut Microbiomes of the Eastern Oyster (Crassostrea virginica) from Coastal Louisiana, USA. PLoS One 7: e51475.

Lebeis, S.L., Paredes, S.H., Lundberg, D.S., Breakfield, N., Gehring, J., McDonald, M., et al. (2015) Salicylic acid modulates colonization of the root microbiome by specific bacterial taxa. Science (80-. ). 349: 860-864.

Ley, R.E., Lozupone, C.A., Hamady, M., Knight, R., and Gordon, J.I. (2008) Worlds within worlds: Evolution of the vertebrate gut microbiota. Nat. Rev. Microbiol. 6: 776-788.

Lokmer, A. and Mathias Wegner, K. (2015) Hemolymph microbiome of Pacific oysters in response to temperature, temperature stress and infection. ISME J. 9: 670-682.

Maansson, M., Vynne, N.G., Klitgaard, A., Nybo, J.L., Melchiorsen, J., Nguyen, D.D., et al. (2016) An Integrated Metabolomic and Genomic Mining Workflow To Uncover the Biosynthetic Potential of Bacteria. mSystems 1: e00028-15. 
Machado, H., Sonnenschein, E.C., Melchiorsen, J., and Gram, L. (2015) Genome mining reveals unlocked bioactive potential of marine Gram-negative bacteria. BMC Genomics 16: 158.

Martens, T., Heidorn, T., Pukall, R., Simon, M., Tindall, B.J., and Brinkhoff, T. (2006) Reclassification of Roseobacter gallaeciensis Ruiz-Ponte et al. 1998 as Phaeobacter gallaeciensis gen. nov., comb. nov., description of Phaeobacter inhibens sp. nov., reclassification of Ruegeria algicola (Lafay et al. 1995) Uchino et al. 1999 as Marinovum algicola gen. nov., comb. nov., and emended descriptions of the genera Roseobacter, Ruegeria and Leisingera. Int. J. Syst. Evol. Microbiol. 56: 1293-1304.

Murphy, B., Jensen, P., and Fenical, W. (2012) The chemistry of marine bacteria. In, Fattorusso,E., Gerwick,W., and Taglialatela-Scafati,O. (eds), Handbook of Marine Natural Products. Springer New York, pp. 153-190.

Newton, R.J., Griffin, L.E., Bowles, K.M., Meile, C., Gifford, S., Givens, C.E., et al. (2010) Genome characteristics of a generalist marine bacterial lineage. ISME J. 4: 784-798.

Ossai, S., Ramachandran, P., Ottesen, A., Reed, E., DePaola, A., and Parveen, S. (2017)

Microbiomes of American Oysters (Crassostrea virginica) Harvested from Two Sites in the Chesapeake Bay. Genome Announc. 5:.

Pohlner, M., Degenhardt, J., von Hoyningen-Huene, A.J.E., Wemheuer, B., Erlmann, N., Schnetger, B., et al. (2017) The Biogeographical Distribution of Benthic Roseobacter Group Members along a Pacific Transect Is Structured by Nutrient Availability within the Sediments and Primary Production in Different Oceanic Provinces. Front. Microbiol. 8: 2550.

Porsby, C.H. and Gram, L. (2016) Phaeobacter inhibens as biocontrol agent against Vibrio vulnificus in oyster models. Food Microbiol. 57: 63-70. 
Porsby, C.H., Nielsen, K.F., and Gram, L. (2008) Phaeobacter and Ruegeria species of the Roseobacter clade colonize separate niches in a Danish Turbot (Scophthalmus maximus)rearing farm and antagonize Vibrio anguillarum under different growth conditions. Appl. Environ. Microbiol. 74: 7356-64.

Porsby, C.H., Webber, M. a, Nielsen, K.F., Piddock, L.J. V, and Gram, L. (2011) Resistance and tolerance to tropodithietic acid, an antimicrobial in aquaculture, is hard to select. Antimicrob. Agents Chemother. 55: 1332-7.

Prado, S., Montes, J., Romalde, J.L., and Barja, J.L. (2009) Inhibitory activity of Phaeobacter strains against aquaculture pathogenic bacteria. Int. Microbiol. 12: 107-114.

Pusztahelyi, T., Holb, I.J., and Pócsi, I. (2015) Secondary metabolites in fungus-plant interactions. Front. Plant Sci. 6: 573.

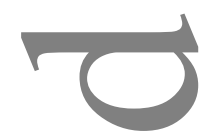

Rao, D., Webb, J.S., and Kjelleberg, S. (2005) Competitive interactions in mixed-species biofilms containing the marine bacterium Pseudoalteromonas tunicata. Appl. Environ. Microbiol. 71: 1729-1736.
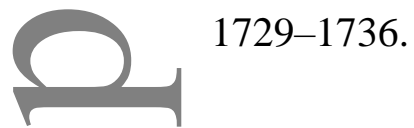

()

Rasmussen, B., Nielsen, K., Machado, H., Melchiorsen, J., Gram, L., and Sonnenschein, E. (2014) Global and Phylogenetic Distribution of Quorum Sensing Signals, Acyl Homoserine Lactones, in the Family of Vibrionaceae. Mar. Drugs 12: 5527-5546.

Rasmussen, B.B., Erner, K.E., Bentzon-Tilia, M., and Gram, L. (2018) Effect of TDA-producing Phaeobacter inhibens on the fish pathogen Vibrio anguillarum in non-axenic algae and copepod systems. Microb. Biotechnol. 0: 1-10.

Rasmussen, B.B., Grotkjær, T., D’Alvise, P.W., Yin, G., Zhang, F., Bunk, B., et al. (2016) Vibrio anguillarum is genetically and phenotypically unaffected by long-term continuous exposure to 
the antibacterial compound tropodithietic acid. Appl. Environ. Microbiol.

Ruiz-Ponte, C., Cilia, V., Lambert, C., and Nicolas, J.L. (1998) Roseobacter gallaeciensis sp. nov., a new marine bacterium isolated from rearings and collectors of the scallop Pecten maximus. Int. J. Syst. Bacteriol. 48: 537-542.

Segev, E., Castañeda, I.S., Sikes, E.L., Vlamakis, H., and Kolter, R. (2016) Bacterial influence on alkenones in live microalgae. J. Phycol. 52: 125-130.

Segev, E., Wyche, T.P., Kim, K.H., Petersen, J., Ellebrandt, C., Vlamakis, H., et al. (2016) Dynamic metabolic exchange governs a marine algal-bacterial interaction. Elife 5:.

Seyedsayamdost, M.R., Case, R.J., Kolter, R., and Clardy, J. (2011) The Jekyll-and-Hyde chemistry of Phaeobacter gallaeciensis. Nat. Chem. 3: 331-335.

Simon, M., Scheuner, C., Meier-Kolthoff, J.P., Brinkhoff, T., Wagner-Döbler, I., Ulbrich, M., et al. (2017) Phylogenomics of Rhodobacteraceae reveals evolutionary adaptation to marine and non-marine habitats. ISME J. 11: 1483-1499.

nnenschein, E.C., Nielsen, K.F., D’Alvise, P., Porsby, C.H., Melchiorsen, J., Heilmann, J., et al. (2017) Global occurrence and heterogeneity of the Roseobacter-clade species Ruegeria mobilis. ISME J. 11: 569-583.

Sonnenschein, E.C., Phippen, C.B.W., Bentzon-Tilia, M., Rasmussen, S.A., Nielsen, K.F., and Gram, L. (2018) Phylogenetic distribution of roseobacticides in the Roseobacter group and their effect on microalgae. Environ. Microbiol. Rep. 10: 383-393.

Sonnenschein, E.C., Stierhof, M., Goralczyk, S., Vabre, F.M., Pellissier, L., Hanssen, K.Ø., et al. (2017) Pseudochelin A, a siderophore of Pseudoalteromonas piscicida S2040. Tetrahedron 73: 
2633-2637.

Vezzulli, L., Stagnaro, L., Grande, C., Tassistro, G., Canesi, L., and Pruzzo, C. (2018) Comparative 16S rDNA Gene-Based Microbiota Profiles of the Pacific Oyster (Crassostrea gigas) and the Mediterranean Mussel (Mytilus galloprovincialis) from a Shellfish Farm (Ligurian Sea, Italy). Microb. Ecol. 75: 495-504.

Wegner, K., Volkenborn, N., Peter, H., and Eiler, A. (2013) Disturbance induced decoupling between host genetics and composition of the associated microbiome. BMC Microbiol. 13: 252.

West, N.J., Obernosterer, I., Zemb, O., and Lebaron, P. (2008) Major differences of bacterial diversity and activity inside and outside of a natural iron-fertilized phytoplankton bloom in the Southern Ocean. Environ. Microbiol. 10: 738-756.

\footnotetext{
(Aietz, M., Gram, L., Jørgensen, B., and Schramm, A. (2010) Latitudinal patterns in the abundance of major marine bacterioplankton groups. Aquat. Microb. Ecol. 61: 179-189.

Wilson, M.Z., Wang, R., Gitai, Z., and Seyedsayamdost, M.R. (2016) Mode of action and resistance (3) studies unveil new roles for tropodithietic acid as an anticancer agent and the $\gamma$-glutamyl cycle as a proton sink. Proc. Natl. Acad. Sci. 113: 1630-1635.

Zabeti, N., Bonin, P., Volkman, J.K., Guasco, S., and Rontani, J.-F. (2010) Fatty acid composition of bacterial strains associated with living cells of the haptophyte Emiliania huxleyi. Org. Geochem. 41: 627-636.

Zubkov, M. V., Fuchs, B.M., Archer, S.D., Kiene, R.P., Amann, R., and Burkill, P.H. (2001) Linking the composition of bacterioplankton to rapid turnover of dissolved dimethylsulphoniopropionate in an algal bloom in the North Sea. Environ. Microbiol. 3: 304- 
311.

\section{Figure legends.}

Figure 1. The composition of bacterial communities associated with Emiliania huxleyi in response to the addition of Phaeobacter inhibens DSM 17395 at 0,24, 48 and 96 hours in duplicates. The compositions of individual microbiomes are illustrated as relative abundances of all the bacterial orders observed in co-cultures of the microalga and different densities of Phaeobacter inhibens DSM 17395 over time. Only orders with abundance above $2 \%$ were included (the remaining low 1 abundance orders are represented by the distance up to 1.00). EH_OTU 4 containing the added $P$. inhibens was removed from the dataset prior to plotting. $\mathrm{T}_{0}$ : untreated time zero control, $\mathrm{C}$ : untreated control, LD: low density $\left(10^{4} \mathrm{CFU} \mathrm{mL}^{-1}\right), \mathrm{HD}$ : high density $\left(10^{6} \mathrm{CFU} \mathrm{mL}^{-1}\right)$.

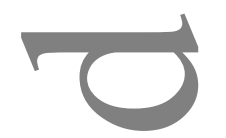

(1) Figure 2. The composition of bacterial communities associated with European flat oysters (Ostrea (Jedulis) in response to the addition of Phaeobacter inhibens DSM 17395 at 0 and 48 hours in triplicates (control) and duplicates (treated). The compositions of individual microbiomes are illustrated as relative abundances of bacterial orders observed in oysters over a time course of $48 \mathrm{~h}$. Only orders with abundance above $2 \%$ were included (the remaining low abundance orders are represented by the distance up to 1.00). OE_OTU 1 containing the added $P$. inhibens was removed from the dataset prior to plotting. Some oyster received a high density (HD) of Phaeobacter inhibens DSM $17395\left(10^{7} \mathrm{CFU} \mathrm{mL}^{-1}\right)$ while others were untreated (controls; C).

Figure 3. Richness of bacterial microbiomes observed in A) Emiliania huxleyi (microalga) and B) Ostrea edulis (European flat oysters) in response to the addition of Phaeobacter inhibens DSM 
17395. The richness is depicted as the average Chao1 richness estimate, error bars represent the standard deviation of the average. OE_OTU 1 and EH_OTU 4 containing the added $P$. inhibens were removed from the datasets prior to plotting. $\mathrm{T}_{0}$ : untreated time zero control, $\mathrm{C}$ : untreated control, LD: low density, HD: high density. Note the difference in the y-axis. Statistical significance of the change in the oyster microbiomes $(\mathrm{P} \leq 0.05)$ is indicated by an asterisk.

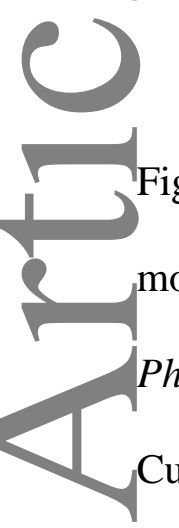

Figure 4. Heatmap indicating the $\log _{10}(\mathrm{x}+1)$ transformed relative abundances of sequences of the 40 most abundant OTUs of the Emiliania huxleyi microbiome in response to the addition of Phaeobacter inhibens DSM 17395. Individual microbiomes are arranged according to the BrayCurtis distances between samples as indicated by the tree above the heatmaps (tree not drawn to scale). The SILVA annotation with identity scores (\%) are listed next to the individual OTU. OTUs, which were unclassified at genus level, were listed with their nearest classified level (family, order (1) or class level).

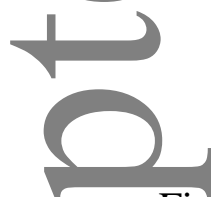

Figure 5. Heatmap indicating the $\log _{10}(x+1)$ transformed relative abundances of sequences of the 40 ) most abundant OTUs of the Ostrea edulis microbiome in response to the addition of Phaeobacter inhibens DSM 17395. Individual microbiomes are arranged according to the Bray-Curtis distances between samples as indicated by the tree above the heatmaps (tree not drawn to scale). The SILVA annotation with identity scores (\%) are listed next to the individual OTU. OTUs, which were unclassified at genus level, were listed with their nearest classified level (family, order or class level). OTUs 7, 29, and 12 were only classified as "Bacteria" according to the SILVA database and thus, the most significant alignment from NCBI's BLAST has been used instead (GenBank accession number and \% identity is listed). 


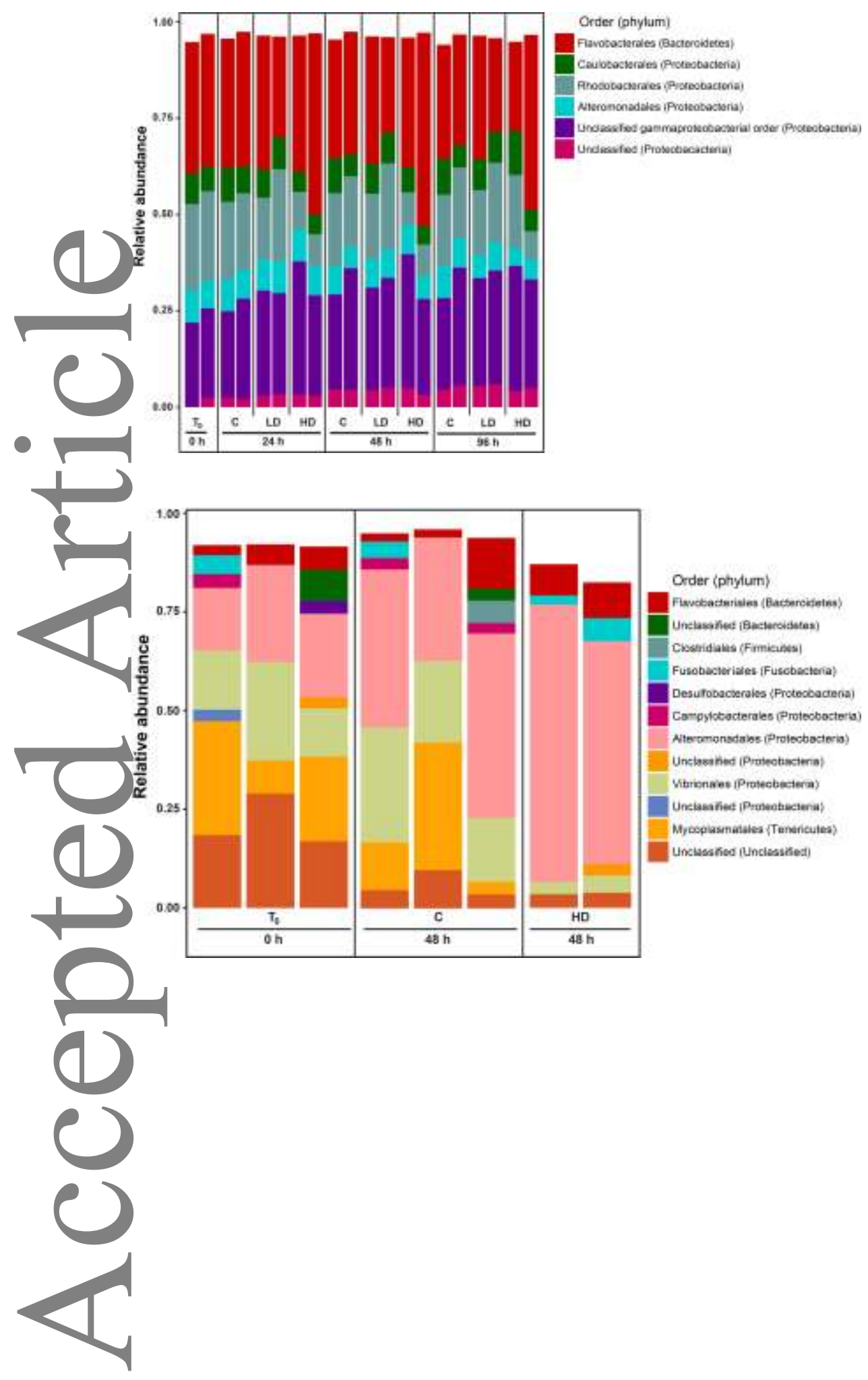

This article is protected by copyright. All rights reserved. 

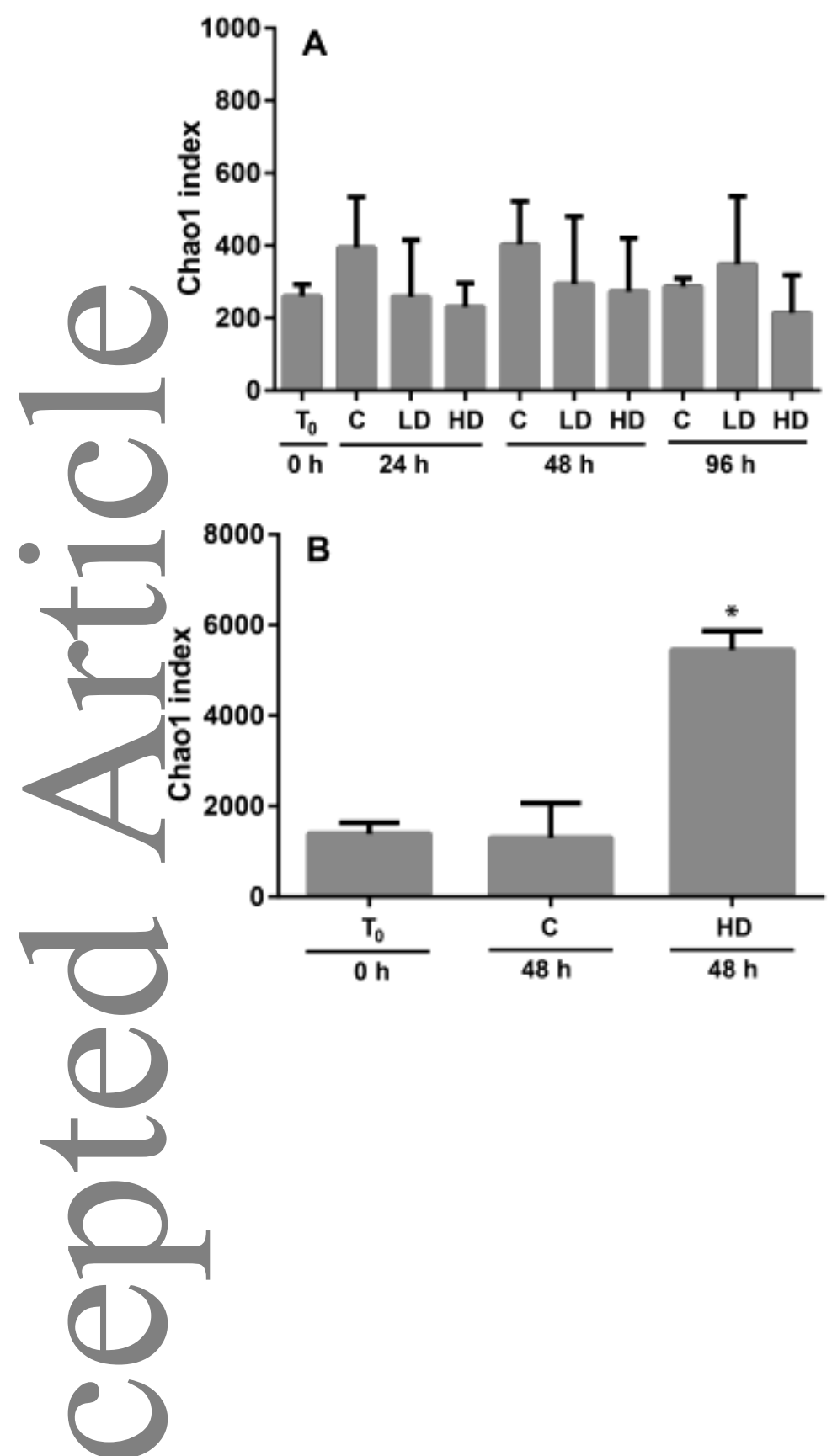

0

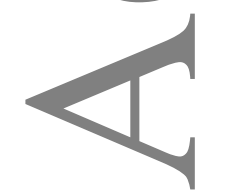




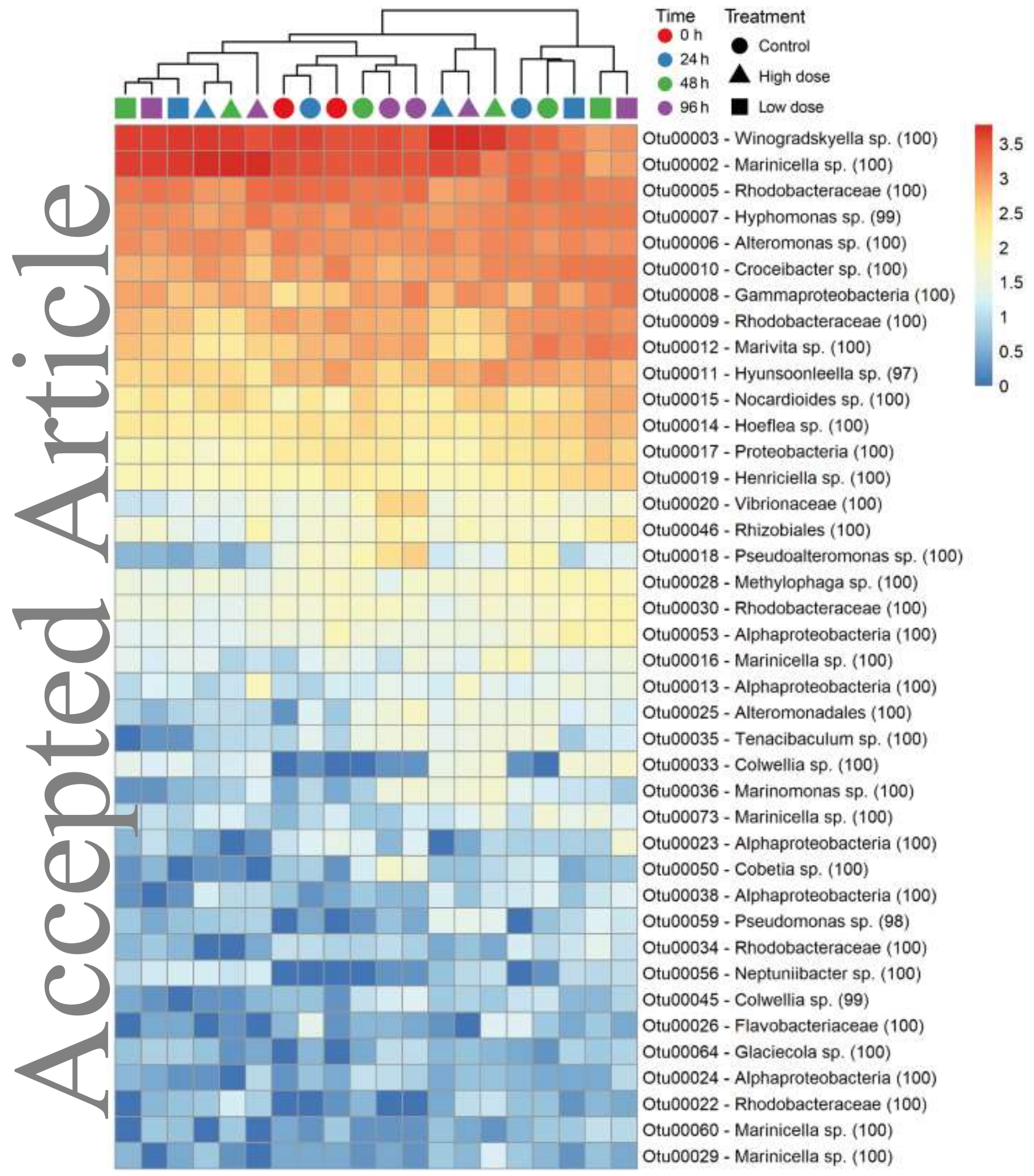

This article is protected by copyright. All rights reserved. 
